International Journal of Applied Mathematical Research, $6(1)(2016) 1-3$
International Journal of Applied Mathematical Research
WPC
Website: www.sciencepubco.com/index.php/IJAMR
doi: $10.14419 /$ ijamr.v6il.6864
Research paper

\title{
Finding a measure, a ring theoretical approach
}

\author{
Ali Parsian \\ Department of Mathematics, Tafresh University, Tafresh 39518 79611, Iran
}

Parsian@Tafreshu.ac.ir

\begin{abstract}
Let $S$ be a nonempty set and $F$ consists of all $Z_{2}$ characteristic functions defined on $S$. We are supposed to introduce a ring isomorphic to $(P(S), \triangle, \cap)$, whose set is $F$. Then, assuming a finitely additive function $m$ defined on $P(S)$, we change $P(S)$ to a pseudometric space $\left(P(S), d_{m}\right)$ in which its pseudometric is defined by $m$. Among other things, we investigate the concepts of convergence and continuity in the induced pseudometric space. Moreover, a theorem on the measure of some kinds of elements in $(P(S), m)$ will be established. At the end, as an application in probability theory, the probability of some events in the space of permutations with uniform probability will be determined. Some illustrative examples are included to show the usefulness and applicability of results.
\end{abstract}

Keywords: Boolean ring, Measure space, Pseudometric space, Uniform probability space.

\section{Introduction}

Some of the propositions in mathematics can be used in science or other branches of mathematics to obtain some new results. For instance, a force field is always a vector field representing forces. The laws of conservation of energy, conservation of angular momentum, Kepler's laws of planetary motion in astronomy are some of the consequences of the mathematical theory of central vector fields [10]. As another example, let us to consider the fundamental theorem of algebra. It states that every non constant single variable polynomial with complex coefficients has at least one complex root. In [2] the authors proved that the Gauss-Bonnet theorem in differential geometry implies the fundamental theorem of algebra. There are also other proofs which involve some analysis, or at least the topological concept of continuity of real or complex functions, differentiable or analytic functions [4], Cauchy's integral theorem [12], or even the theory of compact differentiable manifolds $[3,11]$ and intersection theory mod 2 [9]. Recently, some another proofs about the existence of the roots for polynomials, also has been given in $[5,6]$.

In this paper, as a follow up to the method of mentioned works, we consider the Boolean ring $(P(S), \triangle, \cap)[8]$ with a measure on $P(S)$ and investigate its properties to find a mathematical formula for the measure of some special kinds of subsets of $S$. At the end, we apply the previous work to the theory of uniform probability spaces. We are able to include some basic results whose detailed proofs are not easily available elsewhere.

\section{Preliminary notes}

Let $S$ be a nonempty set. For each $A \subseteq S$, let $\chi_{A}$ be the characteristic function of $A$ with values in $Z_{2}$. i.e., $\chi_{A}(x)=\overline{1}$ for $x \in A$ and $\chi_{A}(x)=$ $\overline{0}$ for $x \notin A$. Let $F=\left\{\chi_{A} \mid A \subseteq S\right\}$, then for $B, C \subseteq S$, the binary operations $\oplus_{S}$ and $\otimes_{S}$ are defined on $F$ by

$\left(\chi_{B} \oplus_{S} \chi_{C}\right)(x)=\chi_{B}(x) \oplus \chi_{C}(x), \quad\left(\chi_{B} \otimes_{S} \chi_{C}\right)(x)=\chi_{B}(x) \otimes \chi_{C}(x)$,

in which $\oplus$ and $\otimes$ denote the operations of addition and multiplication in $Z_{2}$ respectively.

An algebra of sets on $S$ is a nonempty collection $\mathbf{A} \subseteq P(S)$ such that,

$$
\emptyset \in \mathbf{A}, \quad \forall B, C \in \mathbf{A}: B \cup C \in \mathbf{A}, \quad \forall B \in \mathbf{A}: S-B \in \mathbf{A} .
$$

A finitely additive function on $\mathbf{A}$ is a function $m: \mathbf{A} \rightarrow[0,+\infty]$ such that $m(B \cup C)=m(B)+m(C)$ for all $B, C \in \mathbf{A}$ with $B \cap C=\emptyset$ [7] The function $m$ assigns to each element $B$ of $\mathbf{A}$, an element $m(B)$ of the set of non negative extended real numbers. The finitely additive function $m$ is called bounded if $m(S)<+\infty$.

An algebra $\mathbf{A} \subseteq P(S)$ of sets is called a $\sigma$-algebra, if every union of any countable collection of sets in $\mathbf{A}$ is again in $\mathbf{A}$. A countably additive function $m$ on a $\sigma$-algebra $\mathbf{A}$ is a function $m: \mathbf{A} \rightarrow[0,+\infty]$ such that $m\left(\cup A_{n}\right)=\sum m\left(A_{n}\right)$ for each sequence $A_{n}$ of disjoint sets in $\mathbf{A}$. If $m$ is a countably additive function on an algebra $\mathbf{A} \subseteq P(S)$, then $(\mathbf{A}, m)$ is called a measure space. If $(\mathbf{A}, m)$ is a measure space and $m(S)=1$, then $(\mathbf{A}, m)$ is called a probability space.

A pseudometric space is a generalized metric space in which the distance between two distinct points can be zero. A pseudometric on $S$ is a real function $d: S \times S \rightarrow R$ which satisfies the conditions

$$
\forall x, y, z \in S: d(x, y)=d(y, x), d(x, x)=0, d(x, z) \leq d(x, y)+d(y, z)
$$

The pseudometric topology is defined exactly as the metric space topology. A pseudometric topology is Hausdorff if and only if it is a metric space [1].

In the following, we are going to establish the aforementioned structures on $P(S)$. 


\section{Mathematical structures on $P(S)$}

\subsection{Making $P(S)$ to a Boolean ring}

Let $F$ be the set of all $Z_{2}$ characteristic functions of the subsets of $S$. Obviously $\chi_{S}=\overline{1}, \chi_{\emptyset}=0$ and $\chi_{B}=\chi_{C} \Leftrightarrow B=C$. Therefore:

Theorem 3.1.1 The sets $P(S)$ and $F$ are equivalent.

Let $B \triangle C$ denotes the symmetric difference of the sets $B, C$. The proofs of the following two theorems are straightforward.

Theorem 3.1.2 Let $B, C \subseteq S$, then

$\chi_{B} \oplus_{S} \chi_{C}=\chi_{B \triangle C}, \quad \chi_{B} \otimes_{S} \chi_{C}=\chi_{B \cap C}, \quad \chi_{S-B}=\overline{1} \oplus_{S} \chi_{B}$.

Theorem 3.1.3 Let $n \in N$ and $B_{1}, \ldots, B_{n} \subseteq S$, then

$\chi_{B_{1}} \oplus_{S} \ldots \oplus_{S} \chi_{B_{n}}=\chi_{B_{1} \triangle \ldots \triangle B_{n}}, \quad \chi_{B_{1}} \otimes_{S} \ldots \otimes_{S} \chi_{B_{n}}=\chi_{B_{1} \cap \ldots \cap B_{n}}$.

Theorem 3.1.4 The triple $\left(F, \oplus_{S}, \otimes_{S}\right)$ is a Boolean unitary ring. Proof. Let $B \subseteq S$ then $\chi_{B} \otimes \chi_{B}=\chi_{B \cap B}=\chi_{B}$.

Theorem 3.1.5 The rings $(P(S), \triangle, \cap)$ and $\left(F, \oplus_{S}, \otimes_{S}\right)$ are isomorphic.

Proof. The map $\varphi: P(S) \rightarrow F$ defined by $\varphi(A)=\chi_{A}$ is bijective. Moreover, Theorem 3.1.2 implies that

$\varphi(B \triangle C)=\chi_{B \triangle C}=\chi_{B} \oplus_{S} \chi_{C}=\varphi(B) \oplus_{S} \varphi(C)$,

$\varphi(B \cap C)=\chi_{B \cap C}=\chi_{B} \otimes_{S} \chi_{C}=\varphi(B) \otimes_{S} \varphi(C)$.

\subsection{Making $P(S)$ to a measure space}

Let $B \in P(S)$ and $\mu(B)$ is $+\infty$ for an infinite set $B$ and is equal to the number of elements in $B$ for a finite set. Then $\mu$ is a countably additive function defined for all subsets of $S$, therefore:

Theorem 3.2.1 The ordered pair $(P(S), \mu)$ is a measure space.

\subsection{Making $P(S)$ to a pseudometric space}

Let $m$ be a finitely additive function on the algebra $P(S)$. Theorem 3.2.1 shows that such a function exists. For $B, C \subseteq S$ let $d_{m}(B, C)=$ $m(B \triangle C)$, then:

Theorem 3.3.1 The ordered pair $\left(P(S), d_{m}\right)$ is a pseudometric space. Proof. Obviously

$$
d_{m}(B, C)=d_{m}(C, B) \geq 0, \quad B=C \Rightarrow d_{m}(B, C)=0
$$

for all $B, C \subseteq S$. For $B, C, D \subseteq S$ we have

$$
\begin{aligned}
d_{m}(B, D)+d_{m}(D, C) & -d_{m}(B, C) \\
& =m(B \triangle D)+m(D \triangle C)-m(B \triangle C) \\
& =m(B-D)+m(D-B)+m(D-C) \\
& +m(C-D)-m(B-C)-m(C-B) \\
& =[m(B-D)+m(D-C)-m(B-C)] \\
& +[m(C-D)+(D-B)-m(C-B)] \geq 0 .
\end{aligned}
$$

Because

$$
\begin{gathered}
(C-D) \cap(D-B)=(B-D) \cap(D-C)=\emptyset, \\
C-B \subseteq(C-D) \cup(D-B), \quad B-C \subseteq(B-D) \cup(D-C) .
\end{gathered}
$$

This completes the proof.
Let $B \subseteq S$ and $\left\{B_{n}\right\}_{n \in N}$ be a sequence of elements in $(P(S), m)$. Then we write $\lim _{n \rightarrow+\infty} B_{n}=B$ if and only if $\lim _{n \rightarrow+\infty} d_{m}\left(B_{n}, B\right)=0$. As a consequence we have:

Theorem 3.3.2 If $\lim _{n \rightarrow+\infty} B_{n}=B$, then $\lim _{n \rightarrow+\infty} m\left(B_{n}\right)=m(B)$.

Proof. Let $\lim _{n \rightarrow+\infty} B_{n}=B$, then $\lim _{n \rightarrow+\infty} d\left(B_{n}, B\right)=0$, so for any $\varepsilon>0$ there exists $n_{0} \in N$ such that, if $n>n_{0}$ then $m\left(B_{n}-B\right)+m\left(B-B_{n}\right)<\varepsilon$. But

$$
\begin{aligned}
& m\left(B_{n}\right)=m\left(B_{n}-B\right)+m\left(B \cap B_{n}\right) \leq m\left(B_{n}-B\right)+m(B), \\
& m(B)=m\left(B-B_{n}\right)+m\left(B_{n} \cap B\right) \leq m\left(B-B_{n}\right)+m\left(B_{n}\right) .
\end{aligned}
$$

Therefore $\left|m\left(B_{n}\right)-m(B)\right| \leq m\left(B_{n}-B\right)+m\left(B-B_{n}\right)<\varepsilon$ for $n>n_{0}$. This completes the proof.

Theorem 3.3.3 The function $m:\left(P(S), d_{m}\right) \rightarrow(R,||)$ is continuous.

With the preceding notations let $B, C \subseteq S$ are such that $d_{\mu}(B, C)=0$, then $\mu(B \triangle C)=0$. Thus $\mu(B-C)=\mu(C-B)=0$ and $B=C$. Therefore:

Theorem 3.3.4 The ordered pair $\left(P(S), d_{\mu}\right)$ is a metric space.

In the next section, we are going to find the measure of some special kinds of sets in a bounded measure space $(P(S), m)$.

\section{The measure of some sets}

Let $N$ be the set of natural numbers, $O \subseteq N$ be the set of odd numbers and $E=N-O$. For the bounded measure space $(P(S), m)$ and the subsets $B_{1}, \cdots, B_{n}$, let

$$
\begin{array}{r}
O_{n}=\left\{a \in S \mid \exists r \in O, r \leq n, i_{1}, \ldots, i_{r} \leq n, \operatorname{card}\left\{i_{1}, \ldots, i_{r}\right\}=r\right. \\
\left., a \in B_{i_{1}} \cap \ldots \cap B_{i_{r}}\right\}, \\
E_{n}=\left\{a \in S \mid \exists r \in E, r \leq n, i_{1}, \ldots i_{r} \leq n, \operatorname{card}\left\{i_{1}, \ldots, i_{r}\right\}=r\right. \\
\left., a \in B_{i_{1}} \cap \ldots \cap B_{i_{r}}\right\} .
\end{array}
$$

Since $O_{n}$ and $E_{n}$ are disjoint subsets, then $m\left(O_{n}\right)+m\left(E_{n}\right)=m(S)$. In the following Theorem, $O_{n}$ is interpreted due to some operations in commutative unitary ring $(P(S), \triangle, \cap)$. The proof of the Theorem is a consequence of the associativity property of symmetric difference and a straightforward induction.

Theorem 4.1 Let $n \in N$ and $B_{1}, \cdots, B_{n}$ are some subsets of $S$. Then

$O_{n}=B_{1} \triangle \cdots \triangle B_{n}$

The following Theorem is a consequence of Theorem 4.1.

Theorem 4.2 Let $m\left(B_{i}\right)=m_{i}, m\left(B_{i} \cap B_{j}\right)=m_{i j}, m\left(B_{i} \cap B_{j} \cap B_{k}\right)=$ $m_{i j k}, \ldots, S_{1}^{n}=\sum_{i} m_{i}, S_{2}^{n}=\sum_{i<j} m_{i j}, S_{3}^{n}=\sum_{i<j<k} m_{i j k}, \ldots$ for $n \in N$ and $B_{1}, \ldots, B_{n} \in P(S)$. Then $m\left(O_{n}\right)=\sum_{r=1}^{n}(-2)^{r-1} S_{r}^{n}$.

Proof. Let $n=2$, then

$$
\begin{aligned}
m\left(O_{2}\right) & =m\left(B_{1} \triangle B_{2}\right)=m\left(B_{1} \cup B_{2}\right)-m\left(B_{1} \cap B_{2}\right) \\
& =m\left(B_{1}\right)+m\left(B_{2}\right)-2 m\left(B_{1} \cap B_{2}\right)=m_{1}+m_{2}-2 m_{12} \\
& =S_{1}^{2}-2 S_{2}^{2}=\sum_{r=1}^{2}(-2)^{r-1} S_{r}^{2} .
\end{aligned}
$$

Therefore the Theorem is hold for $n=2$. Let $n=k$ and $m\left(O_{k}\right)=$ $\sum_{r=1}^{k}(-2)^{r-1} S_{r}^{k}$. If $C_{i}=B_{i} \cap B_{k+1}$ for $1 \leq i \leq k$, then $m\left(C_{i}\right)=$ $m_{i(k+1)}, m\left(C_{i} \cap C_{j}\right)=m_{i j(k+1)}, \cdots$ and etc. Thus for $n=k+1$ we 
have

$$
\begin{aligned}
m\left(O_{k+1}\right) & =m\left(B_{1} \triangle \cdots \triangle B_{k} \triangle B_{k+1}\right)=m\left[\left(B_{1} \triangle \cdots \triangle B_{k}\right) \triangle B_{k+1}\right] \\
& =m\left(B_{1} \triangle \cdots \triangle B_{k}\right)+m\left(B_{k+1}\right)-2 m\left[\left(B_{1} \triangle \cdots \triangle B_{k}\right) \cap B_{k+1}\right] \\
& =m\left(B_{1} \triangle \cdots \triangle B_{k}\right)+m\left(B_{k+1}\right) \\
& -2 m\left[\left(B_{1} \cap B_{k+1}\right) \triangle \cdots \triangle\left(B_{k} \cap B_{k+1}\right)\right] \\
& =m\left(O_{k}\right)+m\left(B_{k+1}\right)-2 m\left(C_{1} \triangle \cdots \triangle C_{k}\right) \\
& =\sum_{r=1}^{k}(-2)^{r-1} S_{r}^{k}+m_{k+1}-2 \sum_{l=1}^{k}(-2)^{l-1} T_{l}^{k},
\end{aligned}
$$

for which we have $T_{l}^{k}=\sum_{i} m\left(C_{i}\right)=\sum_{i<k+1} m_{i(k+1)}, \quad T_{2}=$ $\sum_{i<j} m\left(C_{i} \cap C_{j}\right)=\sum_{i<j<k+1} m_{i j(k+1)}, \cdots$ and etc. Thus

$$
\begin{aligned}
m\left(O_{k+1}\right) & =\sum_{r=1}^{k}(-2)^{r-1} S_{r}^{k}+m_{k+1}+\sum_{l=1}^{k}(-2)^{l} T_{l}^{k} \\
& =\left(S_{1}^{k}+m_{k+1}\right)-2\left(S_{2}^{k}+T_{1}^{k}\right)+4\left(S_{3}^{k}+T_{2}^{k}\right)-\cdots \\
& +(-2)^{k-1}\left(S_{k}^{k}+T_{k-1}^{k}\right)+(-2)^{k} T_{k}^{k}=\sum_{r=1}^{k+1}(-2)^{r-1} S_{r}^{k+1}
\end{aligned}
$$

This completes the proof.

In the following section, we are supposed to present some applications of Theorem 4.2.

\section{Estimation a probability}

In this part we estimate the measure of $O_{n}$ in a uniform discrete probability space [14]. First of all we prove:

Theorem 5.1 If $n \in N$, then $n !>\left(\frac{n}{e}\right)^{n}$.

Proof. If $n=1$ then the inequality is hold. Let the Theorem is true for $n=k$, i.e., $k !>\left(\frac{k}{e}\right)^{k}$. A computation using the inequality of the arithmetic and geometric means shows that the sequence $\{(1+$ $\left.\left.\frac{1}{n}\right)^{n}\right\}_{n \in N}$ is a bounded increasing sequence with limit $e$. Therefore $\left(1+\frac{1}{n}\right)^{n}<e$ for all $n \in N$. If $n=k+1$, then the hypothesis of the induction, and the above inequality implies that

$$
\begin{aligned}
(k+1) !=(k+1) k ! & >(k+1)\left(\frac{k}{e}\right)^{k} \\
& =\left(\frac{k+1}{e}\right)^{k+1} \frac{e}{\left(1+\frac{1}{k}\right)^{k}}>\left(\frac{k+1}{e}\right)^{k+1} .
\end{aligned}
$$

This completes the induction.

Theorem 5.2 Let $S$ be the set of all permutations of the set $M=\{1, \cdots, n\}, a=\frac{1-e^{-2}}{2}$, and $B_{i}=\{f \in S: f(i)=i\}$ for $i=1, \cdots, n$. Then $m\left(O_{n}\right)=\sum_{k=1}^{k=n}(-2)^{k-1}\left(\frac{1}{k !}\right)$, and if $n \geq 10$ then $\left|m\left(O_{n}\right)-a\right|<\frac{2}{1000}$. Moreover, $m\left(O_{n}\right)<\frac{7}{15}$ for $n>4$.

Proof. A computation using uniform probability laws yields that

$m_{i}=\frac{1}{n}, m_{i j}=\frac{1}{n(n-1)}, m_{i j k}=\frac{1}{n(n-1)(n-2)}, \cdots$,

$S_{1}^{n}=\frac{1}{1 !}, S_{2}^{n}=\frac{1}{2 !}, S_{3}^{n}=\frac{1}{3 !}, \ldots$

so Theorem 4.2 implies that $m\left(O_{n}\right)=\sum_{k=1}^{k=n}(-2)^{k-1}\left(\frac{1}{k !}\right)$. Moreover, a computation using Theorem 5.1 shows that for $n \geq 10$ we have

$$
\begin{aligned}
\left|m\left(O_{n}\right)-a\right|=\sum_{k=n}^{+\infty} \frac{(-2)^{k}}{(k+1) !} & <\sum_{k=n}^{+\infty}\left(\frac{2 e}{k+1}\right)^{k} \\
& <\sum_{k=n}^{+\infty} 2^{-k}=2^{-n+1}<\frac{2}{1000}
\end{aligned}
$$

The last inequality in the Theorem, comes from the Leibniz's rule for alternative series [13].
The following examples are some confirmations for the previous Theorem:

Example 5.3 Let $n$ computers with labels $1, \ldots, n$ are used randomly by $n$ users with labels $1, \ldots, n$. Let $1 \leq i \leq n$ and $B_{i}$ be the event that the $i$-th computer is used by the user the same label. The probabilities $m_{i}, m_{i j}, m_{i j k}, \cdots$ are computed as in the proof of Theorem 5.2. Therefore, the results of the previous theorem, also holds in this case. In particular, if the number of users is bigger than 9 , then the probability of the occurrence of an odd number of $B_{i} \mathrm{~s}$ is less than 0.002 . In other words, the probability that an odd (res even) number of computers are used by the same odd (res. even) number of users with same labels randomly, is less (res. greater) than 0.002 (res. 0.980). Moreover

$m\left(O_{1}\right)=1, \quad m\left(O_{2}\right)=0, \quad m\left(O_{3}\right)=\frac{2}{3}$.

Example 5.4 An institute of standards and industrial research dispatches randomly two inspectors to two food manufacturer companies once a season. Obviously, the dispatching of exactly one inspector on his previous visited company is impossible. The previous theorem also implies that

$m\left(O_{2}\right)=1-2(1 / 2 !)=0$.

\section{References}

[1] R. Abraham, J. E. Marsden, T. Ratiu, Manifolds, tensor analysis, and applications, Springer Verlag, (1988).

[2] J. M. Almira, "Yet another application of the Gauss-Bonnet theorem for the sphere", Bulletin of the Belgian Mathematical Society, V(14) (2007), 341-342.

[3] J. M. Almira, A. Romero, "Some Riemannian geometric proofs of the fundamental theorem of algebra", Differential Geometry-Dynamical Systems, V(14), (2012), 1-4.

[4] R. V. Churchill, J. W. Brown, R. F. Verhey, Complex variables and applications, Mc Graw-Hill, (2004).

[5] O. R. B. de Oliveira, "The fundamental theorem of algebra: from the four basic operations", American Mathematical Monthly (119), V(9), (2012), 753-758.

[6] M. Eisermann,"The fundamental theorem of algebra made effective: An elementary real-algebraic proof via Sturm chains", American Math ematical Monthly, (119), V(9), (2012), 715-752.

[7] G. B. Folland, Real analysis, modern techniques and applications, John Wiley \& Sons, Inc., (1999).

[8] S. Givant, P. Halmos, Introduction to Boolean algebra, Springer Verlag, (2009).

[9] V. Guillman, A. Pollack, Differential topology, AMS Chelsea Publish ing, (2010).

[10] M. W. Hirsch, S. Smale, R. L. Devaney, Differential equations, dynamical systems, and an introduction to chaos, Academic Press, (2013).

[11] J. W. Milnor, Topology from the differentiable view point, Princeton University Press, (1997).

[12] A. R. Schep, "A simple complex analysis and an advanced calculus proof of the fundamental theorem of algebra", American Mathematical Monthly, V(116), (2009), 67-68.

[13] J. Stewart, Calculus, Brooks/Cole, (2012).

[14] R. E. Walpole, R. H. Myers, S. L. Myers, K. Ye, Probability and statistics for engineers and scientists, Prentice Hall, (2011). 